\title{
A NOTE ON AMERICAN OPTIONS WITH VARYING EXERCISE PRICE
}

\author{
J. N. DEWYNNE ${ }^{1}$ and P. WILMOTT ${ }^{2}$
}

(Received 15 August 1992, revised 17 August 1993)

\begin{abstract}
We examine the valuation of American options in a discrete time setting where the exercise price is known a priori but varies with time. (This is in contrast with the classical BlackScholes [2] analysis, which lies in a continuous time framework and with constant exercise price.) In particular we consider a time series of exercise prices which are themselves a realisation of the share price random walk - that of the previous year, say.
\end{abstract}

\section{Introduction}

A financial option is the right to buy (or sell) an asset at a specified time in the future (the expiry date, $T$ ) for a known amount (the exercise price, $E$ ). As this is a right and not an obligation, the holder of such a right can only benefit from it. To see this, consider the possible events which can occur at expiry, for an option to buy. If the asset price, $S$, is below the exercise price then the option will not be exercised as the asset can be obtained more cheaply by buying it directly. If the asset price is above the exercise price then the option is worth $S-E$; since the asset may be bought for $E$ and immediately sold for $S$, the positive difference is a riskless profit. Since the holder of an option can only benefit, the option has a positive value and the holder must pay a premium for the option. The problem is how to value an option prior to expiry when the outcome (at expiry) is not known a priori.

The problem for the fair value of an option was first solved by Black and Scholes [2]. They construct a portfolio of one option and a predetermined number of assets which, because of the correlation between the random movements of the asset and option prices, eliminates all the indeterminacy in the change in value of the portfolio.

\footnotetext{
'Department of Mathematics, University of Southampton, Southampton, SO17 1BJ, England.

${ }^{2}$ Mathematical Inst., Oxford University, Oxford, England, and Dept. of Math., Imperial College, London, England.

(C) Australian Mathematical Society, 1995, Serial-fee code 0334-2700/95
} 
(Their means of eliminating risk is known as ' $\Delta$-hedging'.) Their analysis results in the well known Black-Scholes partial differential equation. Other authors have derived valuation formulæ in the context of discrete time and asset price charges. Typically the asset price follows a binomial random walk and changes only across discrete time steps (see, for example, Cox, Ross and Rubinstein [5]). See Hull [14] and Wilmott $e$ t al. [20] for a general introduction to the subject.

The discussion above describes a European call option. The adjective call refers to the right to buy the asset at expiry. It is also possible to purchase put options which give the holder the right to sell the asset. The difference between calls and puts is essentially a difference in the payoff, the value of an option at expiry. The adjective European indicates that the right can only be exercised at expiry. There are also American options which give the holder the right to exercise the option at any time up to and including the expiry date. It is the American feature which is of interest to us here. (We refer the reader to Cox and Rubinstein [6], Hull [14], Dewynne et al. [9] or Wilmott et al. [20] for a more complete discussion of the differences between the valuation of European and American options.) In the Black-Scholes framework, the valuation of an American option is a free boundary problem (see also Dewynne et al. [9]), similar to the 'oxygen consumption problem' (Crank and Gupta [7]). As such, American options are path dependent - if the value of the asset underlying the option crosses an optimal exercise boundary (the free boundary), the option should be exercised immediately and thus cease to exist before expiry (assuming the holder acts optimally).

In this paper we consider the valuation of American put options in a discrete time setting and with an exercise price that varies discretely in time. The life time of the option is divided into $N$ equal discrete time steps of size $\delta \tau$. The payoff for early exercise at a time $n \delta \tau$ is given by

$$
P^{n}(S)=\max \left(E^{n}-S, 0\right),
$$

where $E^{n}$ is the exercise price at time step $n$ and is known in advance. If the option is not exercised before the expiry then its value at expiry is $P^{N}(S)=\max \left(E^{N}-S, 0\right)$. We are particularly interested in a sequence of exercise prices, $\left(E^{n}\right)_{n=1}^{N}$, which cannot be represented by a smooth function. ${ }^{3}$ In our example we take this sequence to be a realisation of a geometric Brownian random walk. We have in mind a situation where the exercise price $E_{n}$ is the price that the underlying asset had, say, a year ago. Thus, although random, the exercise prices are known in advance.

\footnotetext{
${ }^{3}$ When the exercise price is a known smooth function of time then the Black-Scholes continuous time analysis is applicable. In this situation it is well known that the valuation problem may be formulated as a linear complementarity problem (Wilmott et al. [20]) or variational inequality (Jaillet et al. [16]). These formulations, which are independent of the precise form of the payoff function, may be solved numerically by finite difference methods or finite element methods (Wilmott et al. [20]). The standard method is to use the projected-SOR algorithm of Cryer; see Elliott and Ockendon [11] or Crank [8].
} 
In our model, time is taken to be discrete, whereas the asset price can attain any strictly positive value; $S$ is a continuous variable. Our model thus lies between the Black-Scholes [2] (continuous time and asset price) and the Cox, Ross and Rubinstein [5] (discrete time and asset price) models. For completeness we also mention the jump model of, for example, Cox and Ross [3] which is a continuous time and discrete asset model. It is important to distinguish between these models. The total elimination of risk by $\Delta$-hedging is only known to be possible in two cases:

1. in the continuous time, continuous asset price case of Black and Scholes, where we can apply Itô's lemma and

2. the discrete time and asset price binomial model.

In other situations, such as the discrete time trinomial case or the discrete time, continuous asset price situation considered here, we cannot eliminate risk totally and are left with a risky portfolio. Thus we must value this residual risk and we discuss this further shortly.

A practical application of our model is in connection with the Retail Price Index (RPI), which is the difference between the price of a basket of goods compared with the price of the same basket one year earlier. A significant proportion of the UK Gross National Product now has its future price rises limited directly with reference to RPI - the 'RPI - $x$ ' formula. This includes, in the UK, British Telecom, British Gas and all the electricity and water companies, for example.

In Section 2, we consider one way of valuing such an option using arguments similar to those of Black and Scholes. This leads us to a difference equation which is inherently unstable and ill-posed. We then consider, in Section 3, a related model which leads to a well-posed valuation problem for the option. Finally, in Section 4, we present a numerical example.

\section{i. An unstable model}

We begin with an assumption about the random nature of asset prices. The commonest and simplest assumption is that the asset price $S$ follows a geometric Brownian motion given by the stochastic differential equation

$$
d S=\mu S d t+\sigma S d X
$$

where $\mu$ and $\sigma$ are constants (the underlying growth and standard deviation respectively) and $d X$ is a Wiener process, see $\emptyset \mathrm{ksendal}$ [18], with $\mathscr{E}[d X]=0$ and $\mathscr{E}\left[d X^{2}\right]=d t$. Here $\mathscr{E}$ is the expectation operator. A discrete time version of this continuous random walk is

$$
\delta S=\mu S \delta t+\sigma S \phi \sqrt{\delta t}
$$


where $\delta t$ is a fixed, small time-step, $\phi$ is a random variable drawn from a standardised normal distribution (zero mean and unit variance) and $\delta S$ is the jump in the asset price. Time increases in fixed steps $\delta t$, but $\delta S$ and hence $S$ can take any real value. Note that $S$ has the fractal property of being self-similar over time scales much larger than $\delta t$ (Peters [19], Chapter 8).

With the notation $V^{n}(S)$ as the value of the put when the price of the underlying asset is $S$ and the time is $n \delta t$, and the expiry date denoted by $n=N$, we now address the problem of valuing our put option. We first follow the ideas of Black and Scholes [2] and attempt to construct a risk free portfolio consisting of one such option and a number of assets, $\Delta^{n}$. Note that $\Delta^{n}$ is fixed from time $n \delta t$ to $(n+1) \delta t$. We write the value of this portfolio as $\Pi^{n}$, so that

$$
\Pi^{n}=V^{n}+\Delta^{n} S .
$$

This is the value of the portfolio at time $n \delta t$. At time $(n+1) \delta t$ the value of the assets will have changed to $S+\delta S$, with $\delta S$ unknown a priori, and hence the value of the option will have changed to $V^{n+1}(S+\delta S)$. The number of assets owned will be the same as at time $n \delta t$. The change in the value of the portfolio $\delta \Pi$ is

$$
\delta \Pi^{n}=V^{n+1}(S+\delta S)+\Delta^{n} \delta S-V^{n} .
$$

For the rest of this section we assume that $V^{n}$ has everywhere a well-defined second derivative with respect to $S$; this will lead to inconsistencies within the model, but the ideas which occur as a consequence of the analysis are indispensable when we come to construct a consistent model.

Supposing that $V^{n+1}$ has two derivatives with respect to $S$, we may use a stochastic Taylor series expansion to show that

$$
\delta \Pi^{n}=V^{n+1}(S)+\delta S V_{S}^{n+1}(S)+\frac{1}{2} \sigma^{2} S^{2} \phi^{2} \delta t V_{S S}^{n+1}(S)-V^{n}(S)+\Delta^{n} \delta S+\mathrm{O}\left(\delta t^{3 / 2}\right) .
$$

We see that the choice

$$
\Delta^{n}=-V_{S}^{n+1}(S)
$$

leads to the elimination, to leading order, of the random component of $d \Pi^{n}$ (the $\phi$ contained within $\delta S$ ). This is the concept of $\Delta$-hedging. ${ }^{4}$

If our model was a continuous time model, we could apply Itô's lemma (see $\varnothing \mathrm{ksendal}[18]$ ) to (4), effectively replacing $\phi^{2}$ by its expected value, 1 . Such a portfolio would be wholly riskless and its return could be set equal to that from a risk-free deposit. This would result in the Black-Scholes equation. Here, however, the portfolio is not entirely riskless; it contains a random $\phi^{2}$ term as well as the smaller terms of $\mathrm{O}\left((\delta t)^{3 / 2}\right)$ that we are going to ignore. This residual risk must be valued. We

${ }^{4}$ Note that $\Delta^{n}$ is the derivative of $V$ at the current asset price but the next time-step. 
make the common assumption that the owner of the option is risk-neutral and expects to make as much from this portfolio as if the cash equivalent had been invested in a riskless account. Thus we set

$$
\mathscr{E}\left[\delta \Pi^{n}\right]=r \Pi^{n} \delta t,
$$

where $r$ is the risk free interest rate. Bringing together (3), (5), (6) and (7), we find that

$$
(1+r \delta t) V^{n}=V^{n+1}+\delta t\left(\frac{1}{2} \sigma^{2} S^{2} V_{s S}^{n+1}+r S V_{s}^{n+1}\right)+\mathrm{O}\left(\delta t^{3 / 2}\right) .
$$

Henceforth we assume that $\delta t$ is sufficiently small, compared to the overall life of the option, for terms of $\mathrm{O}\left(\delta t^{3 / 2}\right)$ to be negligible. In practice, this means that we assume terms of $\mathrm{O}\left(\delta t^{3 / 2}\right)$ are of the same or smaller order of magnitude as other effects that we have deemed negligible, for example, transaction costs (Leland [17], Hodges and Neuberger [13], Dewynne et al. [10]) and the nonconstant volatiles (Black [2]).

The equation bears similarities to the Black-Scholes partial differential equation. Consider the limit $\delta t \rightarrow 0$. We can write $V^{n+1}-V^{n}=V_{t}(S, t) \delta t$ and, on dividing throughout by $\delta t,(8)$ becomes identical to the Black-Scholes equation,

$$
V_{t}+\frac{\sigma^{2} S^{2}}{2} V_{S S}+r S V_{S}-r V=0
$$

We do not take this limit and, indeed, when we come to consider American options we shall see that no sensible limit exists.

Note that (8) is a difference equation for $V^{n}$ given $V^{n+1}$. No boundary conditions are required in $S$ since there are no $S$ derivatives of $V^{n}$. Provided that $V^{n+1}$ has finite $S$-derivatives, it is clear that

$$
V^{n}(0)=\frac{V^{n+1}(0)}{1+r \delta t},
$$

and that, if $V^{n+1}(\infty)=0$, then $V^{n}(\infty)=0$. This gives a recipe for calculating the value of an option given some final data, that is, the payoff at $n=N$. Now we examine the effect of variable exercise price and early exercise.

In Figure 1 we see a schematic representation of the naive method of valuation proposed. We see $V^{n}$ which can, in principle, be evaluated from $V^{n+1}$. Also shown is the payoff at time $n$ given by $\max \left(E^{n}-S, 0\right)$, that is, the payoff for a put option with exercise price $E^{n}$. Observe that for $S<S_{f}^{n}$ we have implied that $V^{n}(S)<$ $\max \left(E^{n}-S, 0\right) .^{5}$

In a case where early exercise is permitted and the value of the option is less than the payoff, an arbitrager would purchase this option, immediately exercise it and sell

\footnotetext{
${ }^{5}$ We have implied here that there is only a single free boundary. In fact, there may be no free boundary. There may, however, be at most one free boundary. We justify this comment in the following section.
} 


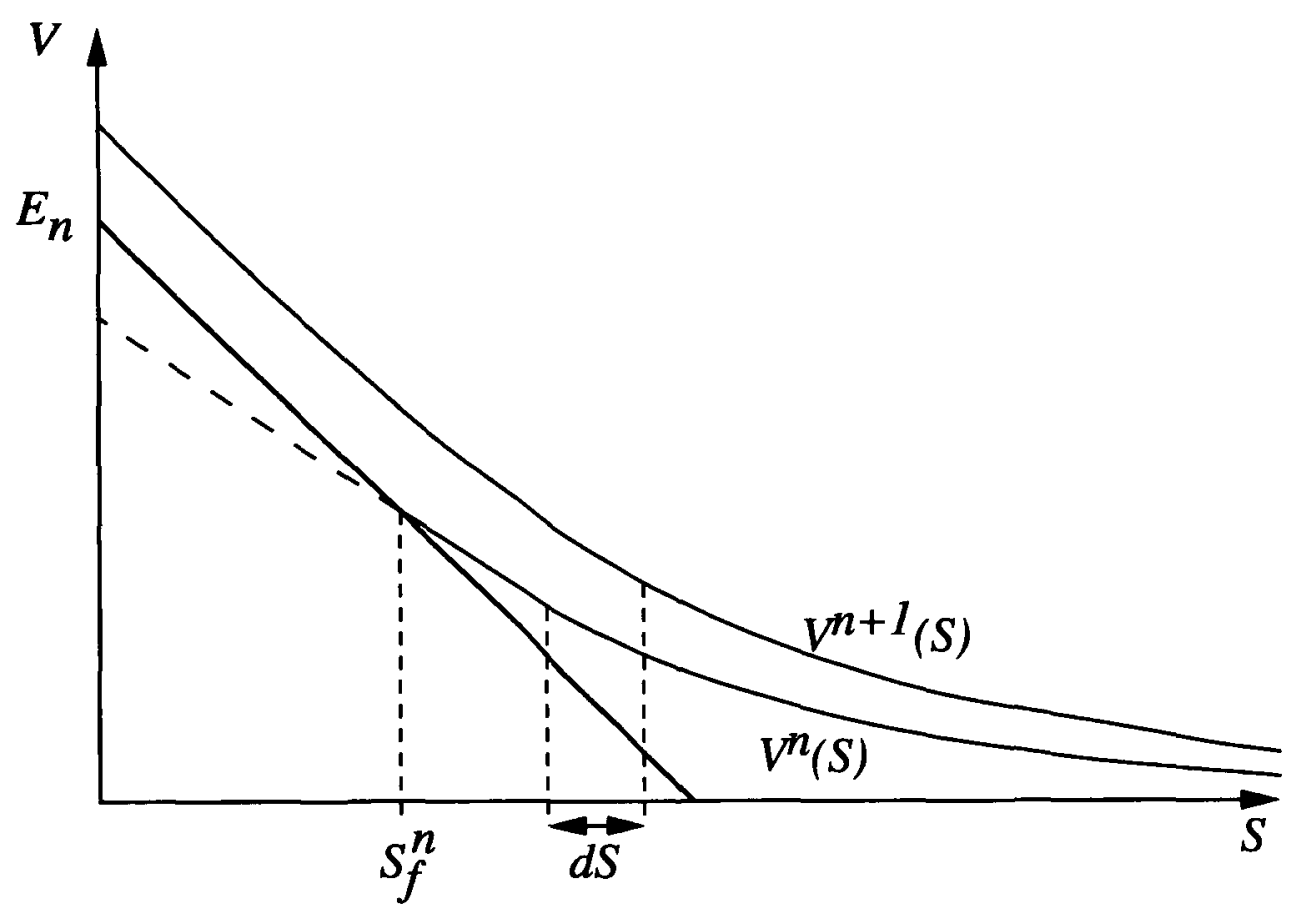

FIGURE 1. A schematic diagram showing $V^{n}(S), V^{n+1}(S), E^{n}$ and $\delta S$.

the assets, thus realising a riskless profit. Such an opportunity would not last and thus it must be the case that

$$
V^{n}(S) \geq \max \left(E^{n}-S, 0\right) .
$$

Our algorithm for valuation is now amended slightly so that we first compute $U^{n}(S)$ as

$$
(1+r \delta t) U^{n}=V^{n+1}+\delta t\left(\frac{1}{2} \sigma^{2} S^{2} V_{S S}^{n+1}+r S V_{S}^{n+1}\right),
$$

and our option value at time step $n$ is given by $V^{n}=\max \left(U^{n}(S), E^{n}-S\right)$. We call this the cut-off procedure. Again it is instructive to note how this differs from the continuous Black-Scholes limit. Suppose that in the Black-Scholes continuous world we have a constant exercise price. The above arbitrage criterion (10) is then of the form

$$
V(S, t) \geq \max (E-S, 0) .
$$


The American option valuation problem may thus be interpreted as an obstacle problem, very similar to the 'oxygen consumption problem' (Crank and Gupta [7]). This is a free boundary problem and in order to find the location of the free boundary $S_{f}(t)$ we require a second boundary condition (at $S=S_{f}(t)$ ) (see Dewynne et al. [9], Jacka [15] or Wilmott $e t$ al. [20]). In fact, $V(S, t)$ must meet the payoff function, $\max (S-E, 0)$, smoothly so that the gradient is continuous (see for example Dewynne et al. [9] or Wilmott et al. [20]).

In the case we are considering, as mentioned above, we have no freedom to prescribe the boundary conditions hence we advocate the cut-off procedure. We do note, however, that the hedging argument used in deriving (8) is not valid in an $\mathrm{O}\left(\delta t^{1 / 2}\right)$ neighbourhood of $S=S_{f}^{n+1}$ since $V^{n+1}(S)$ does not have a well defined first or second $S$ derivative there. We discuss this shortly but we next demonstrate a flaw in the model presented in this section which has important consequences.

The change of variable $S=e^{x}$ reduces (8) to a constant coefficient linear diffusionreaction equation. We temporarily ignore $V_{x}^{n+1}$ terms in the resulting equation and set $r$ to zero. Our simplified equation then takes the form

$$
V^{n}=V^{n+1}+\frac{1}{2} \sigma^{2} V_{x x}^{n+1} \delta t .
$$

We now consider the stability of (12) to a small oscillatory perturbation, that is, we set $V^{n+1}=\sin \lambda x$. We find that

$$
V^{n}=\left(1-\frac{1}{2} \sigma^{2} \delta t \lambda^{2}\right) \sin \lambda x .
$$

Thus a perturbation will grow if $\lambda^{2}>4 /\left(\sigma^{2} \delta t\right)$. We conclude that our model is unstable to small wavelength perturbations. The natural $S$-scale for the growing oscillations is $\mathrm{O}\left((\sigma \delta t)^{1 / 2}\right)$. This is exactly the magnitude of the jump in asset price in a time $\delta t$. The consequence of this instability is to make hedging, for which we require $V^{n+1}$ to have a local Taylor series expansion, an impossibility. Our original assumption that $V^{n}$ has a Taylor series expansion does not hold and we must discard our model in its present form. This analysis can be repeated with the first derivative terms retained and $r$ nonzero, but the conclusion is the same.

\section{A stable model}

In the previous section, our differential-difference equation model breaks down because of short wavelength instabilities. We now attempt to regularise the model.

First, let us comment on the work of Harrison and Pliska [12] in a continuous time setting. They show that when an option can be hedged the value of an option is the same as the discounted present value of the expected payoff under the modified 
risk-neutral random walk

$$
d S=r S d t+\sigma S d X
$$

It follows, as can be seen from the Black-Scholes equation, that in continuous time the option value is independent of $\mu$. Our differential-difference equation is also independent of $\mu$; the approximate $\Delta$-hedging has eliminated the parameter $\mu$ to leading order, although it does appear in the small terms we have neglected.

In a discrete time and continuous asset price model there may not be a unique fair value for the price of an option. If, however, we follow the $\Delta$-hedging policy $\Delta=-V_{s}^{n+1}$ then we eliminate the dependence on $\mu$. We therefore adopt the valuation policy based on the insight of Harrison and Pliska [12]: to determine $V^{n}$ from $V^{n+1}$ we calculate the expected value of $V^{n+1}$ given $S$ at time $n$ and then take the maximum of its discounted present value and its payoff if exercised early.

Since the asset price follows a lognormal random walk, the probability density function for the future distribution of $S^{\prime}$ (at time $\delta t$ in the future) is

$$
\frac{1}{\sqrt{2 \pi \delta t} \sigma} \exp \left(-\frac{1}{2 \sigma^{2} \delta t}\left(\log \left(S^{\prime} / S\right)-\left(r-\frac{\sigma^{2}}{2}\right) \delta t\right)^{2}\right) \frac{1}{S^{\prime}}
$$

if $S^{\prime}>0$ and zero otherwise, where $S$ is the current value of the asset price. Thus, in this model, the value $V^{n}(S)$ (if the option is not exercised at time step $n \delta t$ ) is the discounted conditional expectation under the "risk-neutral"density (13) of $V^{n+1}(S)$ given that the asset price at time $n \delta t$ is $S$, that is,

$$
V^{n}(S)=\frac{e^{-r \delta t}}{\sigma \sqrt{2 \pi \delta t}} \int_{0}^{\infty} V^{n+1}\left(S^{\prime}\right) \exp \left(-\frac{1}{2 \sigma^{2} \delta t}\left(\log \left(S^{\prime} / S\right)-\left(r-\frac{\sigma^{2}}{2}\right) \delta t\right)^{2}\right) \frac{d S^{\prime}}{S^{\prime}}
$$

The smoothing which is achieved by this integral version of the valuation recipe ensures that the solution is stable. If the option is exercised at time $n \delta t$ then

$$
V^{n}(S)=E^{n}-S
$$

and we take the value of the option to be the maximum of the two values (14) and (15). Clearly, if the value given by (14) exceeds that given by (15) the option should be held or sold, and if the value given by (15) exceeds that given by (14), the option should be exercised.

Note that (14) is equivalent to solving the (continuous time) Black-Scholes [2] partial differential equation, (9), from time $(n+1) \delta t$ to $n \delta t$ with final data $V^{n+1}(S)$ $S \geq 0$. Insisting on a nonsingular solution is equivalent to the boundary conditions $V_{t}^{n}(0)=r V^{n}(0)$ (see (9) and note that $V(0, t)$ remaining bounded is equivalent to $S^{2} V_{S S} \rightarrow 0$ and $S V_{S} \rightarrow 0$ as $S \rightarrow 0$ ) and $V^{n}(\infty)=0$. Solving (9) numerically is 
in fact simpler than evaluating (14) numerically. Since we intend solving our model numerically, we choose to solve (9). ${ }^{6}$

As before, in order to eliminate arbitrage possibilities, we must insist that the value $V^{n}(S)$ is greater than or equal to the payoff function and must be cut off as necessary. This will generally result in discontinuities in $V_{S}^{n}$ which would not be sustainable in continuous time but which are allowed (and inevitable) in discrete time. Our American option valuation is therefore, strictly speaking, no longer a free boundary problem since our formulation (14) does not permit us to prescribe any boundary conditions. We end this section with a suggestion for the name for the type of option which we are discussing here. In the same spirit which names options on interest rate caps as 'captions' and options on interest rate swaps as 'swaptions' we will name our options - whose payoff depends on a fractal time series - 'fractions'.

\section{Discussion of the model and an example}

As an example, in this section we choose a sequence of exercise prices $E^{n}$ which is a realisation of the asset price over some prior period. This sequence is known beforehand and we take the volatility, $\sigma$ for the asset price's present random walk to be the same as that calculated from the prior period.

Since the standard deviation for $E^{n+1}-E^{n}$ is $\sigma \delta t^{1 / 2}$ over a period of order $\delta t$, the motion of the payoff 'obstacle' is greater in magnitude than the change in $V^{n}$ before we apply the cut off (which is $O(\delta t)$ ). We also apply the cut-off condition

$$
V^{n}(0)=\max \left(E^{n}, e^{-r \delta t} V^{n+1}(0)\right) .
$$

If $V^{n}(0)=E^{n}$ then the cut-off condition has come into effect and there will be a 'free' boundary', at $S=S_{f}^{n}$. Although the point $S_{f}^{n}$ is found from the cut-off condition we continue to call it a free boundary since, if it exists, it can only be determined by solving the problem. If $V^{n}(0)>E^{n}$ then the option value lies above the payoff and there is no free boundary. We conclude that the free boundary need not move

\footnotetext{
${ }^{6}$ Note that, in principle, it is possible to give an analytic expression for the value of this option, since its valuation consists in a sequence of calculations of discounted expected values and application of a cut-off procedure. Given that the payoff is known for expiry and that the sequence $E^{n}$ is given in advance, it is clearly possible to time-step back from the payoff by applying (14) and then taking the maximum of (14) and (15) at each step, providing a new 'payoff 'for the previous time-step. Such an analytical procedure will, in general, lead to an unmanageable number of quadratures.

${ }^{7}$ The free boundary, if it exists, is unique. This follows from (14) and the fact, noted above, that $V^{n}$ may be found by solving the Black-Scholes equation with $V^{n+1}$ as final data. The Black-Scholes equation is backward parabolic, may be reduced to the heat equation and therefore satisfies the maximum principle. Thus, since $-V_{S}$ also satisfies (a modified) Black-Scholes equation, it follows that $V_{S} \leq 1$ always as $V_{S} \leq 1$ finally (and also after a cut-off has been applied).
} 
continuously but may appear and disappear as $n$ varies depending only upon the size and direction of the jumps in $E^{n}$. This is illustrated in our example. Note also that the time series $V^{n}(0)$ can be determined immediately from the sequence $E^{n}$ starting with $V^{N}(0)=E^{N}$ without having to solve for $V^{n}(S)$ for $S>0$.

Although we choose to step backward in time discretely via a solution of a partial differential equation rather than using the integral representation (14), we note that there is no sensible limiting problem as $\delta t \rightarrow 0$. We cannot go to the limit $\delta t \rightarrow 0$ because of the cut-off constraint - the magnitude of the jumps in the height of the obstacle are $\mathrm{O}\left(\delta t^{1 / 2}\right)$ and so swamp the natural jumps in $V^{n}$ which are $\mathrm{O}(\delta t)$, for fixed $S$. Our choice of a finite-difference scheme necessarily requires the use of a truncated grid and application of a boundary condition at some maximum value $S_{m}$. We take this boundary condition to be $V^{n}\left(S_{m}\right)=0$ for all times $n \delta t$. We also note that this introduces errors into the numerical solution, but these errors can be made arbitrarily small (for finite values of $\sigma^{2} T$, where $T$ is the expiry date of the option) by taking a sufficiently large value of $S_{m}{ }^{8}$

The Black-Scholes equation (9) becomes a constant coefficient, linear parabolic equation under the transformation $S=E e^{x}$ where $E$ is some typical value of the time series $E^{n}$. We make this transformation and solve the resulting equation using a standard implicit finite difference scheme. We then test for early exercise and apply the cut-off if necessary.

In Figure 2, we show the results for our model for a sequence of exercise prices which are taken from the daily closing prices of the Financial Times Stock Exchange 100 (FTSE 100) index from the six months before October $19^{t h}, 1987$, to six months after $(N=252)$. The FTSE 100 indices have been scaled so that the maximum value of $E^{n}$ is 1 . The volatility during this period is 0.281556 and this is taken to be the value for $\sigma$. The interest rate is taken to be 0.09 p.a.

The figure shows the series $E^{n}(\cdot), V^{n}(0)(0)$ and $S_{f}^{n}(\mathrm{x})$. When $V^{n}(0)>E^{n}$ there is no free boundary. Otherwise $S_{f}^{n}$ must be found as part of the solution of the problem. Observe the large regions where there is no free boundary and the option is held regardless of the asset price. Within the regions where early exercise is optimal there are smaller and smaller regions where the free boundary disappears, and where it is therefore optimal to retain the option until a later date. An obvious comparison to make is with a Cantor set - here, however, the fractal nature of the early exercise

\footnotetext{
${ }^{8}$ As we know the sequence $E^{n}$ and we have a maximum principle for the Black-Scholes equation, it is a relatively simple matter to obtain an upper bound on $V^{n}\left(S_{m}\right)$ and hence on the error introduced by approximating this value by zero. It is then not difficult to obtain a bound on the maximum difference between the solution of the actual problem posed on the positive $S$ axis and the numerical problem solved on the interval $\left[0, S_{m}\right]$. The details are routine.
} 


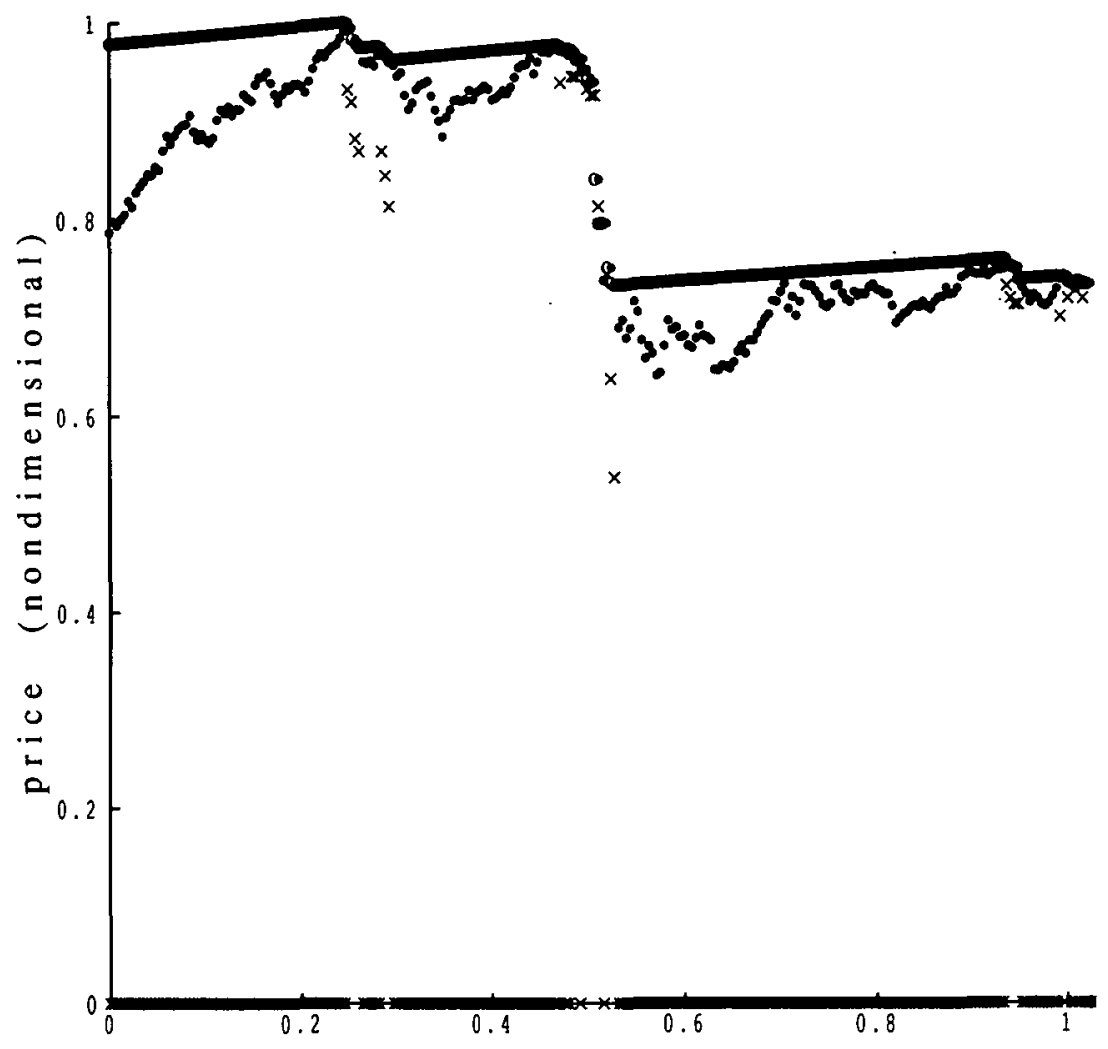

Tim e (years)

FIGURE 2. Results of the model using real data. The series $E^{n}(\cdot), V^{n}(0)(0)$ and $S_{f}^{n}(x)$ are shown. See text for details.

region does not go beyond a finite time-scale (one day, in this example). In Figure 3, we show $V^{n}(S)$ against $S$ at a time during the life of the option when there is a free boundary.

\section{Acknowledgement}

We would like to thank Mark Blundell for his interest in this problem and a referee of an earlier draft of this paper whose comments lead to significant improvements in content and presentation. One of us (P. W.) acknowledges the support of the Royal Society. 


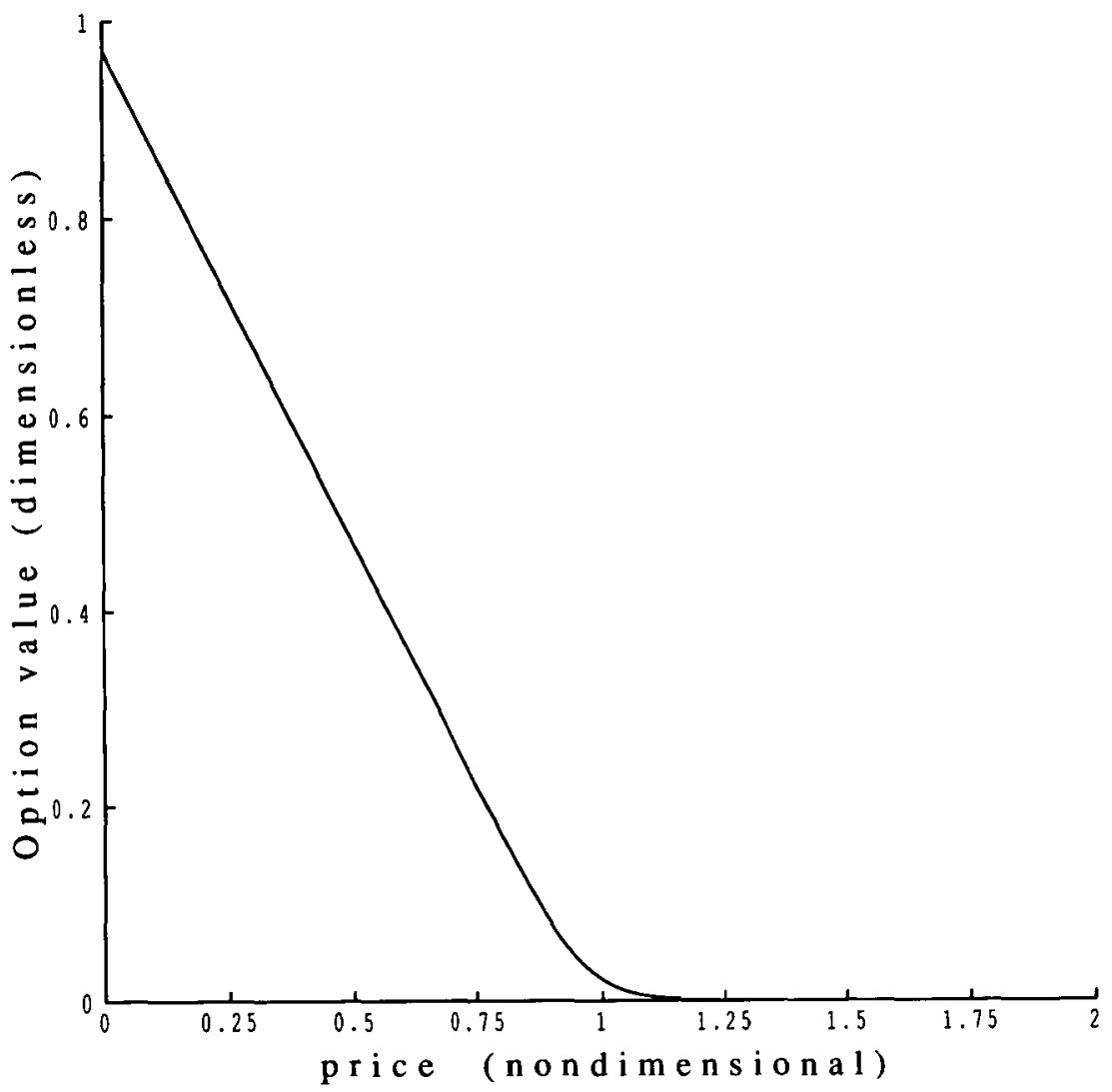

FIGURE 3. The option price, $V^{n}(S)$, vs. $S$ (dimensionless) at $n=128$.

\section{References}

[1] F. Black, "The holes in Black-Scholes", in "From Black-Scholes to black-holes", (Risk/Finex, London, 1992).

[2] F. Black and M. Scholes, "The pricing of options and corporate liabilities", J. Pol. Economy 81 (1973) 637-659.

[3] J. C. Cox and S. A. Ross, "The pricing of options for jump processes", Working paper no. 2-75, Rodney L. White Center for Financial Research, University of Pennsylvania, (1975).

[4] J. C. Cox and S. A. Ross, "The valuation of options for alternative stochastic processes", J. Fin. Econ 3 (1976) 145-166.

[5] J. C. Cox, S. A. Ross and M. Rubinstein, "Option pricing: a simplified approach", J. Fin. Econ (1979) 7 (1979) 229-263.

[6] J. C. Cox and M. Rubinstein, Options markets (Prentice-Hall, Englewood Cliffs, 1985).

[7] J. Crank and R. S. Gupta, "A moving boundary problem arising from the diffusion of oxygen in absorbing tissue", J. Inst. Maths. Applic. 10 (1972) 19-33.

[8] J. Crank, Free and moving boundary problems (Oxford University Press, Oxford, 1984).

[9] J. N. Dewynne, S. D. Howinson, I. Rupf and P. Wilmott, "Some mathematical results in the pricing 
of American options", Euro. J. Appl. Math. 4 (1993) 381-398.

[10] J. N. Dewynne, A. E. Whalley and P. Wilmott, "Path-dependent options and transaction costs", Phil. Trans. R. Soc. Lond. A 347 (1994) 517-529.

[11] C. M. Elliott and J. R. Ockendon, Weak and variational methods for variational problems (Pitman, Boston, 1982).

[12] J. M. Harrison and S. R. Pliska, "Martingales and stochastic integrals in the theory of continuous trading", Stoch. Proc. Appl. 11 (1981) 261-271.

[13] S. D. Hodges and A. Neuberger, "Optimal replication of contingent claims under transaction costs", Review of Futures Markets 8 (1989).

[14] J. Hull, Options, futures and other derivative securities (2nd Ed.), (Prentice-Hall, Englewood Cliffs, 1993).

[15] S. D. Jacka, “Optimal stopping and the American put", Math. Finan. 1 (1991) 1-14.

[16] P. Jaillet, D. Lamberton and B. Lapeyre, "Variational inequalities and the pricing of American options", Acta Applicandae Mathematica (1990).

[17] H. E. Leland, "Option pricing and replication with transaction costs", Journal of Finance 15 (1985) 1283-1295.

[18] B. Øksendal, Stochastic differential equations (Springer, 1992).

[19] E. E. Peters, Chaos and order in the capital markets (Wiley, New York, 1991).

[20] P. Wilmott, J. N. Dewynne and S. D. Howinson, Option pricing: mathematical models and computation (Oxford Financial Press, Oxford, 1993). 